\title{
The role of Compton heating in cluster cooling flows
}

\author{
L. Ciotti* J.P. Ostriker ${ }^{\dagger}$ and S. Pellegrini* \\ *Astronomy Department, Bologna University, Italy \\ ${ }^{\dagger} I o A$, Cambridge, UK and Dept. of Astrophys. Sciences, Princeton University, NJ, USA
}

\begin{abstract}
Recent observations by Chandra and XMM-Newton demonstrate that the central gas in "cooling flow" galaxy clusters has a mass cooling rate that decreases rapidly with decreasing temperature. This contrasts the predictions of a steady state cooling flow model. On the basis of these observational results, the gas can be in a steady state only if a steady temperature dependent heating mechanism is present; alternatively the gas could be in an unsteady state, i.e., heated intermittently. Intermittent heating can be produced by accretion on the supermassive black hole residing in the central cluster galaxy, via Compton heating. This mechanism can be effective provided that the radiation temperature of the emitted spectrum is higher than the gas temperature. Here we explore whether this heating mechanism can be at the origin of the enigmatic behavior of the hot gas in the central regions of "cooling flow" clusters. Although several characteristics of Compton heating appear attractive in this respect, we find that the fraction of absorbed heating for realistic gas and radiation temperatures falls short by two orders of magnitude of the required heating.
\end{abstract}

\section{INTRODUCTION}

$\mathrm{X}$-ray observations of the central regions of a large fraction of galaxy clusters prior to Chandra and XMM - Newton were interpreted in terms of cooling and condensing of the intracluster medium (ICM), leading to a subsonic, steady central inflow called cooling flow (Cowie \& Binney 1977, Fabian \& Nulsen 1977). The measurements of radiative cooling times lower than a Hubble time within a cooling radius $\left(r_{\text {cool }}\right)$ of $\lesssim$ few hundreds of kiloparsecs were at the basis of this idea. However, cool gas in the cluster cores (in the form of massive-star formation or formation of low-mass stars, optical emission line nebulae or cold gas) has never been discovered in a quantity large enough to fit with the steady state cooling flow predictions (e.g., Donahue \& Voit 2003). Moreover recent observations from the Chandra and XMM-Newton satellites have ruled out the simple steady state cooling flow model (e.g., Molendi \& Pizzolato 2001, Peterson et al. 2003). In this model one expects emission from gas over the entire temperature range (from $T_{\max }$, the ambient ICM temperature, down to temperature values at which the ICM is undetectable in the X-rays), with the same mass cooling rate $\dot{M}$ at each temperature. The strengths of a few observed emission lines reveal how much gas cools through each temperature, and high resolution spectroscopic observations now show a deficit of emission relative to the cooling flow predictions from gas below $\sim T_{\max } / 3$ (Peterson et al. 2003). In addition, the spectra show increasingly less emission at lower temperatures than the cooling flow model would predict. Empirically, the differential luminosity distribution $\Delta L / \Delta T \propto T^{1 \div 2}$, instead of being temperature-independent as expected in 
the radiative cooling flow model. Lower spectral resolution observations, as from the Chandra ACIS-S detector, also suggest significantly lower mass cooling rates than obtained from previous analyses of ROSAT and ASCA data (McNamara et al. 2000).

These observational results have produced an important astrophysical puzzle and a number of ideas have been suggested to solve it, but none has been proven conclusive yet. For example, cooling may be opposed by heating (Soker et al. 2001) or thermal conduction may suppress cooling (Ruskowkski \& Begelman 2002). 'Cooling flows' generally have embedded non-thermal radio sources and this association suggests that feedback from an AGN may help suppress the cooling of the ICM. This feedback may come from the impact of the radio jets on the cooling gas (Reynolds et al. 2002, Omma et al. 2003) or from mixing and turbulent heating as buoyant radio plasma rises through the ICM (Brüggen \& Kaiser 2002). Another plausible (intermittent) heating source could be Compton heating resulting from accretion of the cooling gas on the central supermassive black hole of the central galaxy in the cluster core. Although the gas over the body of the galaxy is optically thin, numerical simulations showed that this mechanism is very effective in heating the interstellar medium of giant elliptical galaxies (Ciotti \& Ostriker 2001). Here we explore whether it can provide effective feedback also in the case of cluster cores.

The bolometric luminosity associated with accretion in the galactic nucleus is $L_{\mathrm{BH}}=$ $\varepsilon \dot{M}_{\mathrm{BH}} c^{2}$, where $\dot{M}_{\mathrm{BH}}$ is the accretion rate on the black hole, $\varepsilon$ is the accretion efficiency (usually spanning the range $0.001 \lesssim \varepsilon \lesssim 0.1$ ) and $c$ is the speed of light. Taking $\dot{M}_{\mathrm{BH}}$ of the order of few $10 \mathrm{~s}$ of $M_{\odot} \mathrm{yr}^{-1}$, as suggested by the most recent estimates of the mass accretion rate in 'cooling flows' (Peterson et al. 2003), the power available for heating of the ICM turns out to be $\sim 10^{47} \mathrm{erg} \mathrm{s}^{-1}$. The power required to balance the cooling of the ICM in the 'cooling flow' region must be of the order of its observed X-ray luminosity; this goes from $\sim 10^{43} \mathrm{erg} \mathrm{s}^{-1}$ in the Virgo cluster up to $\sim 10^{45} \mathrm{erg} \mathrm{s}^{-1}$ in the most massive clusters like A1835. Therefore it seems that during the phases of accretion there could be enough power to balance the cooling. The problem is: How much of $L_{\mathrm{BH}}$ is actually trapped by the inflowing gas and therefore is effectively available for its heating? It is clear that for extremely low opacities $L_{\mathrm{BH}}$ would be unable to affect the flow. In the following we estimate how much of $L_{\mathrm{BH}}$ is absorbed by the ICM in the core regions of galaxy clusters, for typical temperature and density profiles.

\section{SETTING THE PROBLEM}

The number of photon-electron interactions per unit volume and in the time interval $\Delta t$ at any radius $r$ in a plasma can be written as:

$$
N_{\gamma \mathrm{e}}(v, r)=N_{\gamma}(v, r) \times \frac{\sigma_{\mathrm{KN}}(v) n_{\mathrm{e}}(r)}{4 \pi r^{2}}=\frac{L_{\mathrm{BH}}(v, r) \Delta t}{h v} \times \frac{\sigma_{\mathrm{KN}}(v) n_{\mathrm{e}}(r)}{4 \pi r^{2}},
$$

where $n_{\mathrm{e}}(r)$ is the electron number density, $h$ is the Planck constant and $\sigma_{\mathrm{KN}}$ is the Klein-Nishina electron scattering cross-section (Lang 1980):

$$
\sigma_{\mathrm{KN}}(v)=\frac{3 \sigma_{\mathrm{T}}}{4}\left\{\frac{1+x}{x^{2}}\left[\frac{2(1+x)}{1+2 x}-\frac{\ln (1+2 x)}{x}\right]+\frac{\ln (1+2 x)}{2 x}-\frac{1+3 x}{(1+2 x)^{2}}\right\},
$$


where $x \equiv v / v_{\mathrm{T}}, v_{\mathrm{T}} \equiv m_{\mathrm{e}} c^{2} / h$ is the Thomson frequency, $m_{\mathrm{e}}$ is the electron mass and $\sigma_{\mathrm{T}}$ is the Thomson cross section. Here for simplicity we assume a gray absorption, i.e.:

$$
L_{\mathrm{BH}}(v, r)=f_{\mathrm{BH}}(v) \times L_{\mathrm{BH}}(r), \quad \int_{0}^{\infty} f_{\mathrm{BH}}(v) d v=1,
$$

where $L_{\mathrm{BH}}(r)$ is the bolometric accretion luminosity that reaches the radius $r$ from the nucleus.

The gas Compton heating (or cooling) per unit frequency at radius $r$ is given by $\Delta E=-N_{\gamma \mathrm{e}}(v) \Delta E_{\gamma}(v, T)$, where $\Delta E$ is the internal energy per unit volume gained (or lost) by the gas from radiation at frequency $v$, and $\Delta E_{\gamma}$ is the energy variation of a photon of frequency $v$ interacting with an electron of gas at temperature $T$. A simple approximation for the energy transfer factor is ${ }^{1}$ :

$$
\Delta E_{\gamma}(v, T)=\frac{x\left(1+3 x^{2} / 8\right)}{1+x^{3}} 4 k_{\mathrm{B}} T-\frac{x^{2}\left(1+x^{2}\right)}{1+x^{3}} m_{\mathrm{e}} c^{2}
$$

where $k_{\mathrm{B}}$ is the Boltzmann constant. After substitution of (1) and (4) in the expression for the Compton heating, and integration over all frequencies, one has:

$$
\frac{\Delta E}{\Delta t}=-\frac{n_{\mathrm{e}}(r)}{n_{\mathrm{t}}(r)} \frac{E(r)}{4 \pi r^{2}} \frac{L_{\mathrm{BH}}(r)}{m_{\mathrm{e}} c^{2}} \frac{8 \Gamma_{\mathrm{C}}}{3}\left[1-\frac{T_{\mathrm{C}}}{T(r)}\right],
$$

where $n_{\mathrm{t}}(r)$ is the total number density,

$$
\Gamma_{\mathrm{C}} \equiv \int_{0}^{\infty} \frac{\left(1+3 x^{2} / 8\right) f_{\mathrm{BH}}(v) \sigma_{\mathrm{KN}}(v)}{1+x^{3}} d v
$$

and the spectral temperature $T_{\mathrm{C}}$ is given by:

$$
T_{\mathrm{C}} \equiv \frac{m_{\mathrm{e}} c^{2}}{4 k_{\mathrm{B}} \Gamma_{\mathrm{C}}} \int_{0}^{\infty} \frac{x\left(1+x^{2}\right) f_{\mathrm{BH}}(v) \sigma_{\mathrm{KN}}(v)}{1+x^{3}} d v
$$

where $m_{\mathrm{e}} c^{2} / 4 k_{\mathrm{B}}=1.48 \times 10^{9} \mathrm{~K}$.

Integrating on $r$ the equation of energy conservation $\partial L_{\mathrm{BH}}(r) / \partial r=-4 \pi r^{2} \partial E(r) / \partial t$, one has

$$
L_{\mathrm{BH}}(r)=L_{\mathrm{BH}}(0) \exp \left\{\frac{8}{3} \frac{\Gamma_{\mathrm{C}}}{m_{\mathrm{e}} c^{2}} \int_{0}^{r} E(r) \frac{n_{\mathrm{e}}(r)}{n_{\mathrm{t}}(r)}\left(1-\frac{T_{\mathrm{C}}}{T(r)}\right) d r\right\} .
$$

From the above equation we can compute the amount of energy actually trapped to heat the gas, for any gas temperature and density profile and any spectral energy distribution of the nuclear photons.

\footnotetext{
${ }^{1}$ This formula reproduces the well known relations $\Delta E_{\gamma} \sim 1.5 k_{\mathrm{B}} T-m_{\mathrm{e}} c^{2} x+O(1 / x)$ for relativistic photon energy $\left(h v \gg m_{\mathrm{e}} c^{2}\right)$, and $\Delta E_{\gamma} \sim 4 k_{\mathrm{B}} T x-m_{\mathrm{e}} c^{2} x^{2}+O\left(x^{3}\right)$ in the classical limit $\left(h v \ll m_{\mathrm{e}} c^{2}\right)$.
} 
We assume that the spectral energy distribution $f_{\mathrm{BH}}(v)$ is made of two distinct contributions. The first is a non thermal distribution of total luminosity $L_{X}(v)=f_{X}(v) \times L_{X}$, with

$$
f_{\mathrm{X}}(v)=\frac{\xi_{2}}{\pi v_{\mathrm{T}}} \sin \left[\frac{\pi\left(1-\xi_{1}\right)}{\xi_{2}}\right]\left(\frac{v_{\mathrm{b}}}{v_{\mathrm{T}}}\right)^{\xi_{1}+\xi_{2}-1} \frac{x^{-\xi_{1}}}{\left(v_{\mathrm{b}} / \nu_{\mathrm{T}}\right)^{\xi_{2}}+x^{\xi_{2}}},
$$

where $h v_{\mathrm{b}}$ is the spectrum break energy and $\xi_{1}$ and $\xi_{1}+\xi_{2}$ are the spectral slopes at low and high frequencies. The second is a blackbody distribution $L_{\mathrm{UV}}(v)=f_{\mathrm{UV}}(v) \times L_{\mathrm{UV}}$ at a temperature $T_{\mathrm{UV}}$, with

$$
f_{\mathrm{UV}}(v)=\frac{15 h^{4}}{\pi^{4} k_{\mathrm{B}}^{4} T_{\mathrm{UV}}^{4}} \frac{v^{3}}{\exp \left(h v / k_{\mathrm{B}} T_{\mathrm{UV}}\right)-1} \simeq \frac{8.17 \times 10^{-43}}{T_{\mathrm{UV}}^{4}} \frac{v^{3}}{\exp \left(h v / k_{\mathrm{B}} T_{\mathrm{UV}}\right)-1}(s)
$$

For these distributions $\int_{0}^{\infty} f_{\mathrm{X}}(v) d v=1$ and $\int_{0}^{\infty} f_{\mathrm{UV}}(v) d v=1$. We also assume that $L_{\mathrm{UV}} \equiv \mathscr{R} L_{\mathrm{X}}$, where $\mathscr{R}$ is a dimensionless parameter measuring the relative importance of the "UV bump" with respect to the high energy part of the spectral energy distribution. Following this choice, the frequency distribution of $L_{\mathrm{BH}}$ in (3) can be written as

$$
f_{\mathrm{BH}}(v)=\frac{f_{\mathrm{X}}+\mathscr{R} f_{\mathrm{UV}}}{1+\mathscr{R}},
$$

and $L_{\mathrm{BH}}(0)=L_{\mathrm{X}}+L_{\mathrm{UV}}=(1+\mathscr{R}) L_{\mathrm{X}}$.

\section{THE RESULTS}

We first estimate the dependence of $\Gamma_{\mathrm{C}}$ and $T_{\mathrm{C}}$, i.e., of the integrals in (6) and (7), on the free parameters $T_{\mathrm{UV}}, v_{\mathrm{b}}, \xi_{1}, \xi_{2}$. We adopt $\xi_{1}=0.9$ and $\xi_{2}=0.7$; these values reproduce the observed spectral shapes of the X-ray and $\gamma$-ray emission of AGNs (e.g., Nandra \& Pounds 1994; Lu \& Yu 1999). The coefficient $\Gamma_{C}$ is evaluated numerically by considering the range $0.1 \leq v_{\mathrm{b}} / v_{\mathrm{T}} \leq 10$ for $f_{\mathrm{X}}$ and the classical limit for $f_{\mathrm{BH}}(v)$, i.e., $k_{\mathrm{B}} T_{\mathrm{UV}} \ll h v_{\mathrm{T}}$. We obtain

$$
\Gamma_{\mathrm{C}} \equiv \Gamma_{\mathrm{X}}+\mathscr{R} \Gamma_{\mathrm{UV}} \simeq \frac{0.77 \times\left(v_{\mathrm{b}} / \nu_{\mathrm{T}}\right)^{-0.06}+\mathscr{R}}{1+\mathscr{R}} \times \sigma_{\mathrm{T}}
$$

A similar evaluation of the dimensionless integral on the r.h.s. of (7) gives

$$
T_{\mathrm{C}} \simeq \frac{8.6 \times 10^{7} \times\left(v_{\mathrm{b}} / v_{\mathrm{T}}\right)^{0.196}+\mathscr{R} T_{\mathrm{UV}}}{0.77 \times\left(v_{\mathrm{b}} / v_{\mathrm{T}}\right)^{-0.06}+\mathscr{R}}(K) .
$$

For observed values of $v_{\mathrm{b}} / v_{\mathrm{T}} \sim 0.2$ and $\mathscr{R} \sim 1$ (e.g., Fabian 1996), $T_{\mathrm{C}}=4 \times 10^{7} \mathrm{~K}$ (independently of $T_{\mathrm{UV}}$ ). This value for $T_{\mathrm{C}}$ is very similar to that derived by Sazonov et al. (2003) from composite mean QSOs spectra.

We next performed the integration in (8) to estimate $L_{\mathrm{BH}}(r)-L_{\mathrm{BH}}(0)$, which is the amount of energy actually trapped by the ICM within a radius $r$. The integration was extended out to the 'cooling radius' $\left(r_{\text {cool }}\right)$, within which a 'cooling flow' could develop. 


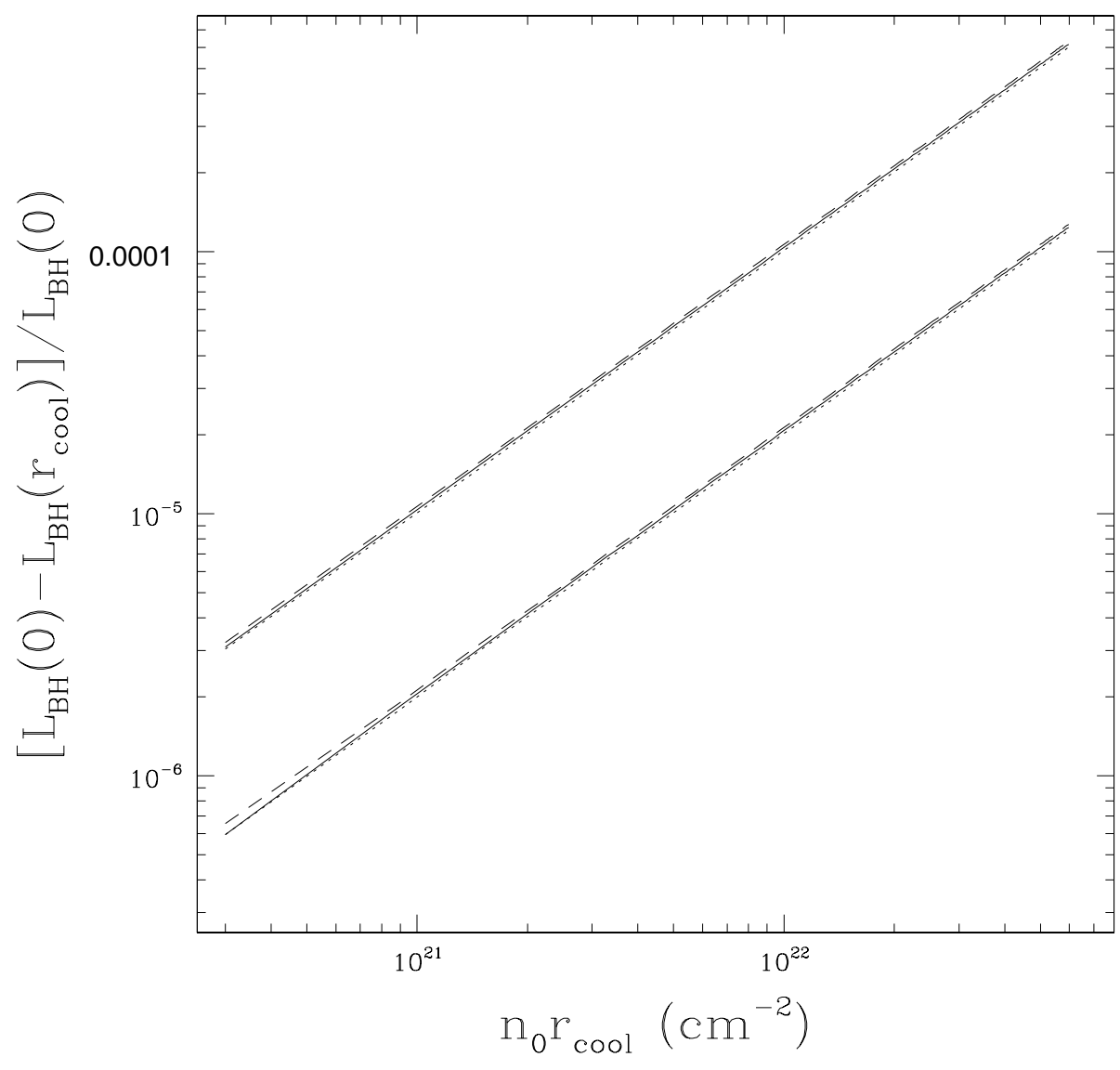

FIGURE 1. The amount of nuclear radiation that actually heats the ICM within $r_{\text {cool }} . v_{\mathrm{b}} / \nu_{\mathrm{T}}$ is fixed at 0.2. The upper set of curves refers to $T_{\mathrm{C}}-T=5 \times 10^{7} \mathrm{~K}$, the lower set to $T_{\mathrm{C}}-T=10^{7} \mathrm{~K}$. Solid lines refer to $\mathscr{R}=1$, dashed ones to $\mathscr{R}=2$ and dotted ones to $\mathscr{R}=0.5$.

For low $T_{\mathrm{C}}$ values $(\sim 1-2 \mathrm{keV}$, as allowed by the study of Sazonov et al. 2003), $r_{\text {cool }}$ encloses the only region within which the ICM temperature $T$ is lower than $T_{\mathrm{C}}$. We assume that the gas is isothermal at a temperature $T$ within $r_{\text {cool }}$. The adopted ICM density profile is a deprojection of the $\beta$-model commonly used to reproduce the observed X-ray surface brightness (i.e., Sarazin 1986): $\rho_{\mathrm{gas}}=\rho_{0}\left[1+\left(r / r_{\mathrm{c}}\right)^{2}\right]^{-3 \beta / 2}$, where $r_{\mathrm{c}}$ is the core radius ( $\sim 250 \mathrm{kpc}$ on average) and $\rho_{0}$ is the total particle density at $r=0$. The integration in (8) gives:

$$
L_{\mathrm{BH}}\left(r_{\text {cool }}\right)=L_{\mathrm{BH}}(0) \exp \left[-\frac{4 \Gamma_{\mathrm{C}}}{m_{\mathrm{e}} c^{2}} \frac{n_{\mathrm{e}}}{n_{\mathrm{t}}} k_{\mathrm{B}}\left(T_{\mathrm{C}}-T\right) n_{0} r_{\mathrm{c}} f\left(\frac{r_{\text {cool }}}{r_{\mathrm{c}}}, \beta\right)\right]
$$

where $n_{0}=\rho_{0} / \mu m_{p}$ with $\mu m_{p}$ the average particle mass. For our choice of $r_{\text {cool }} \leqslant r_{\mathrm{c}}$, the function $f \approx r_{\text {cool }} / r_{\mathrm{c}}$ and is independent of $\beta$. This approximate value for the integral is within $25 \%$ of the true value, for $\beta=0.3-0.9$. In Fig. 1 we plot the amount of nuclear 
radiation that actually heats the ICM within $r_{\text {cool }}$ as a function of $n_{0} r_{\text {cool }}$. The range of variation for $n_{0} r_{\text {cool }}$ is chosen by considering the observed ranges for $r_{\text {cool }}(50-200$ kpc, Peterson et al. 2003) and $n_{0}\left(2 \times 10^{-3}-0.1 \mathrm{~cm}^{-3}\right)$. Taking $n_{\mathrm{e}} / n_{\mathrm{t}}=0.5$, the free parameters left in the expression for $L_{\mathrm{BH}}\left(r_{\text {cool }}\right)$ in (14) are $v_{\mathrm{b}} / \nu_{\mathrm{T}}, \mathscr{R}$ and $T_{\mathrm{C}}-T$. In Fig. 1 we consider a possible range for $\mathscr{R}=0.5-2$ and two extreme cases for the difference $T_{\mathrm{C}}-T$, corresponding to a high $T_{\mathrm{C}}$ value $\left[\sim(5-6) \times 10^{7} \mathrm{~K}\right]$ and a low one $\left(T_{\mathrm{C}} \sim 2 \times 10^{7} \mathrm{~K}\right)$. Note that $\Gamma_{\mathrm{C}}$ varies by $<10 \%$ for $v_{\mathrm{b}} / v_{\mathrm{T}}=0.1-1$, and so the results are totally unaffected by variations of $v_{\mathrm{b}} / \nu_{\mathrm{T}}$ in this range.

\section{CONCLUSIONS}

From Fig. 1 it appears that the power actually available for heating of the ICM within the cooling region goes from few $\times 10^{-6} L_{\mathrm{BH}}(0)$, in the small clusters, up to $10^{-4} L_{\mathrm{BH}}(0)$ in the most massive ones. This makes Compton heating fall short by $\sim$ two orders of magnitude of the required heating and therefore an unplausible mechanism to balance the cooling of the gas in the cluster core, at variance with the situation in elliptical galaxies (Ciotti \& Ostriker 2001).

\section{ACKNOWLEDGMENTS}

L.C. and S.P. thank the IoA for hospitality and financial support during a visit where most of the results presented here were obtained.

\section{REFERENCES}

1. Brüggen, M., Kaiser, C.R. 2002, Nature, 418, 301

2. Ciotti, L., Ostriker, J.P. 2001, Astrophys. J., 551, 131

3. Cowie, L., Binney, J. 1977, Astrophys. J., 215, 723

4. Donahue, M., Voit, M. 2003, astro-ph/0308006

5. Fabian, A.C., Nulsen, P.E.J. 1977, Mon. Not. Roy. Astron. Soc., 180, 479

6. Fabian, A.C. 1996, Proc. 'Roentgenstrahlung from the Universe', eds. Zimmermann, H.U., Truemper, J. and Yorke, H.; MPE Report 263, p. 403-408

7. Lang, K.R. 1980, Astrophysical Formulae, Springer-Verlag Berlin

8. Lu, Y., Yu, Q. 1999, Astrophys. J., 526, L5

9. McNamara, B., et al. 2000, Astrophys. J., 534, L135

10. Molendi, S., Pizzolato, F. 2001, Astrophys. J., 560, 194

11. Nandra, K., Pounds, K.A. 1994, Mon. Not. Roy. Astron. Soc., 268, 405

12. Omma, H., Binney, J., Bryan, G., Slyz, A. 2003, astro-ph/0307471

13. Peterson, J.R., et al. 2003, Astrophys. J., 590, 207

14. Reynolds, C.S., Heinz, S., Begelman, M.C. 2002, Mon. Not. Roy. Astron. Soc., 332, 271

15. Ruskowski, M., Begelman, M.C. 2002, Astrophys. J., 581, 223

16. Sazonov, S.Y., Ostriker, J.P. \& Sunyaev, R.A. 2003

17. Soker, N., White, R.E.III, David, L.P., McNamara, B.R. 2001, Astrophys. J., 549, 832 